\title{
Coexistence of enterotoxigenic Staphylococcus aureus and cytotoxic Clostridium difficile as predisposing factors for septic shock in patients with inflammatory bowel disease
}

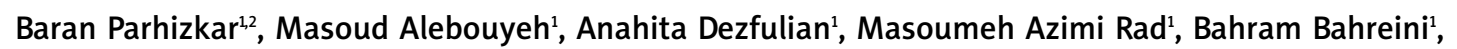 \\ Ehsan Nazemalhosseini ${ }^{1,2}$, Mohammad Mehdi Aslani ${ }^{1}$, Mohammad Reza Zali ${ }^{1,2}$ \\ ${ }^{1}$ Gastroenterology and Liver Diseases Research Center, Shahid Beheshti University of Medical Sciences, \\ Tehran, Iran \\ 2Basic and Molecular Epidemiology of Gastrointestinal Disorders Research Center, Shahid Beheshti University of Medical \\ Sciences, Tehran, Iran
}

Prz Gastroenterol 2013; 8 (3): 206-210 DOI: $10.5114 / p g .2013 .36337$

Key words: Staphylococcus aureus, Clostridium difficile, bacterial superantigens, inflammatory bowel disease, sepsis.

\begin{abstract}
Address for correspondence: Masoud Alebouyeh PhD, Research Center for Gastroenterology and Liver Diseases Research Center, Shahid Beheshti University of Medical Sciences, Tehran, Iran, phone: +98 2122432518, fax: +98 2122432517, e-mail: Masoud.Alebouyeh@gmail.com
\end{abstract}

\begin{abstract}
Superimposed infections in patients with inflammatory bowel disease (IBD) have drawn considerable attention in the disease managements that can lead to increased rates of colectomy and mortality. We followed a case of an IBD patient with severe bloody diarrhea (more than 10 times a day) and diffuse ulceration in his colon that mimicked a flare-up of IBD. Stool examination showed enteric overgrown enterotoxigenic Staphylococcus aureus (sea $a^{+}$together with a low count of cytotoxic Clostridium difficile $\left(t c d B^{+}\right)$bacteria. Antibiotic therapy with oral vancomycin as the sole in vitro determined effective drug normalized the patient's clinical features. The patient died of post-surgical septic shock after colectomy that had been conducted because of deterioration of his clinical state one week after the antibiotic therapy. Sudden release of overgrown intestinal S. aureus superantigens (sea and peptidoglycan) with regard to the cooperative effect of $C$. difficile infection was suspected as a predisposing factor for severe disease.
\end{abstract}

\section{Introduction}

The etiology of inflammatory bowel disease (IBD) is not clear. Multiple causes, including environmental factors and genetic susceptibility, are thought to contribute to deregulated immune functions seen in patients with IBD [1]. Beside genetic and environmental factors, the role of microorganisms in the manifestation of IBD has been discussed in the case of clinical initiation and relapses of IBD [2]. The IBD is a chronic inflammatory condition associated with alteration in immunoregulatory responses by commensal microbiota and their products [1]. The complexity of association between the disease and infections results from variation in immune responses in different conditions. Common and less com- mon intestinal infections are able to trigger IBD. It was shown that enteric infections could be related to the beginning or relapse of IBD. Enteric infections can be responsible for major complications of IBD such as abscesses, bowel obstruction, bowel perforation, colorectal cancer, toxic megacolon, sepsis, and septic shock. While these relationships were reported at the onset of IBD or its relapse, there are few data available to establish the exact manner of these events [3, 4]. Acute and chronic mucosal inflammatory responses in the intestine can promote under specific conditions, such as bacterial overgrowth, release of their toxic proteins or their invasion through a defect in the gastrointestinal tract (GIT) mucosal barrier [5]. Superantigens (SAgs) are microbial 
proteins that are able to trigger large subsets of $\mathrm{T}$ or $\mathrm{B}$ lymphocytes. The cell wall (peptidoglycan) of Gram-positive bacteria, staphylococcal enterotoxins, toxic shock syndrome toxin 1, and streptococcal SAg are examples of T-cell SAgs [6]. The role of bacterial SAgs was shown in different immune stimulatory diseases, such as rheumatoid arthritis, multiple sclerosis, psoriasis, and septic shock [7]. There are some studies reporting an initial contribution of the bacterial SAgs in the pathogenesis of IBD through induction of dysregulated T cell function.

We report a case of lethal septic shock in a patient with IBD who suffered from infective enterocolitis by enterotoxigenic Staphylococcus aureus (S. aureus) and cytotoxic Clostridium difficile (C. difficile) bacteria. Sudden release of overgrown intestinal bacterial SAgs after the therapeutic regime followed by induced immune cell depletion were suspected as causative agents for occurrence of the sepsis at latter stages that led to rapid progression of the disease and death.

\section{Case report}

A 66-year-old man, with a history of IBD since 7 years ago, was admitted to hospital with crampy abdominal pain and frequent urge for defecation. He was under drug treatments for IBD and was stable until 1 month ago, when his condition deteriorated with significant bloody diarrhea, nearly 10 times a day. After admission, treatment with metronidazole and ceftriaxone was started and other medications such as mesalazine $(800 \mathrm{mg}$, three times daily) and azathioprine (50 mg twice daily), which he used before, were continued. Also a stress dose of intravenous glucocorticoids was started. Despite optimal management, he had no clinical response to the treatments. He was febrile (OT $37 / 8$ c), but BP (100/70), PR (95), and RR (18) were normal. Also he had a mild tenderness in the palpation of the lower abdomen; direct rectum examination showed some bloody fecaloid material. Related laboratory criteria included C-reactive protein (CRP) of $63 \mathrm{mg} / \mathrm{l}$, erythrocyte sedimentation rate (ESR) of $39 \mathrm{~mm} / \mathrm{h}$, and white blood cell count (WBC) of $11.1 \times 10^{3} \mu \mathrm{l}$. Stool microscopic examination showed the presence of polymorphonuclears (PMNs) and Gram-positive cocci in high numbers (Figure 1). Stool culture was negative for Campylobacter spp., Shigella spp., Salmonella spp., and Yersinia spp., but positive for $S$. aureus (high count) and C. difficile. The results from hematological and biochemical tests and direct stool exam confirmed the loss of blood in the stool. Liver function tests were normal at the time of admission. PCR results for $S$. aureus enterotoxin A (sea) and $C$. difficile cytotoxin $B(t c d B)$ genes showed specific bands related to these toxins (Figure 2). Elevated values for CRP and ESR and existence of high numbers of WBC in stool samples together with patient history of anti-

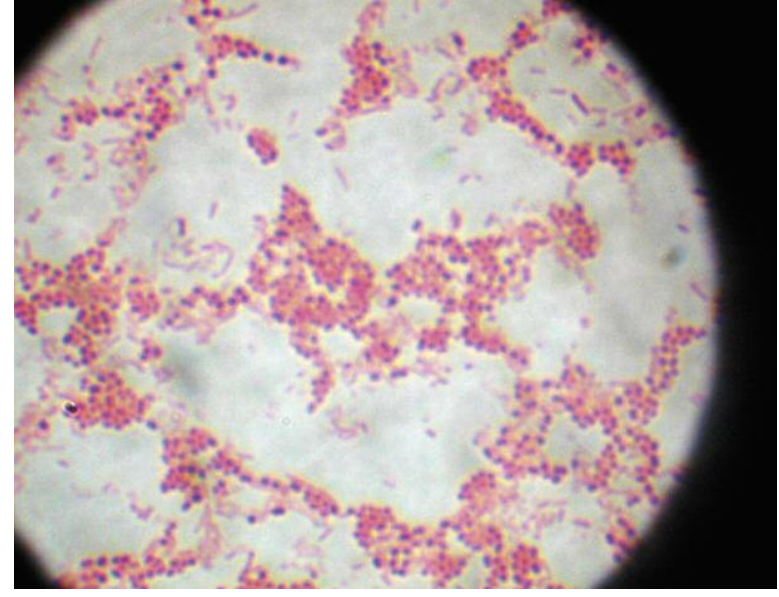

Fig. 1. Microscopic examination of stool sample by Gram staining method. Clusters of Grampositive cocci and bacilli with imbalanced intestinal microbial flora are seen

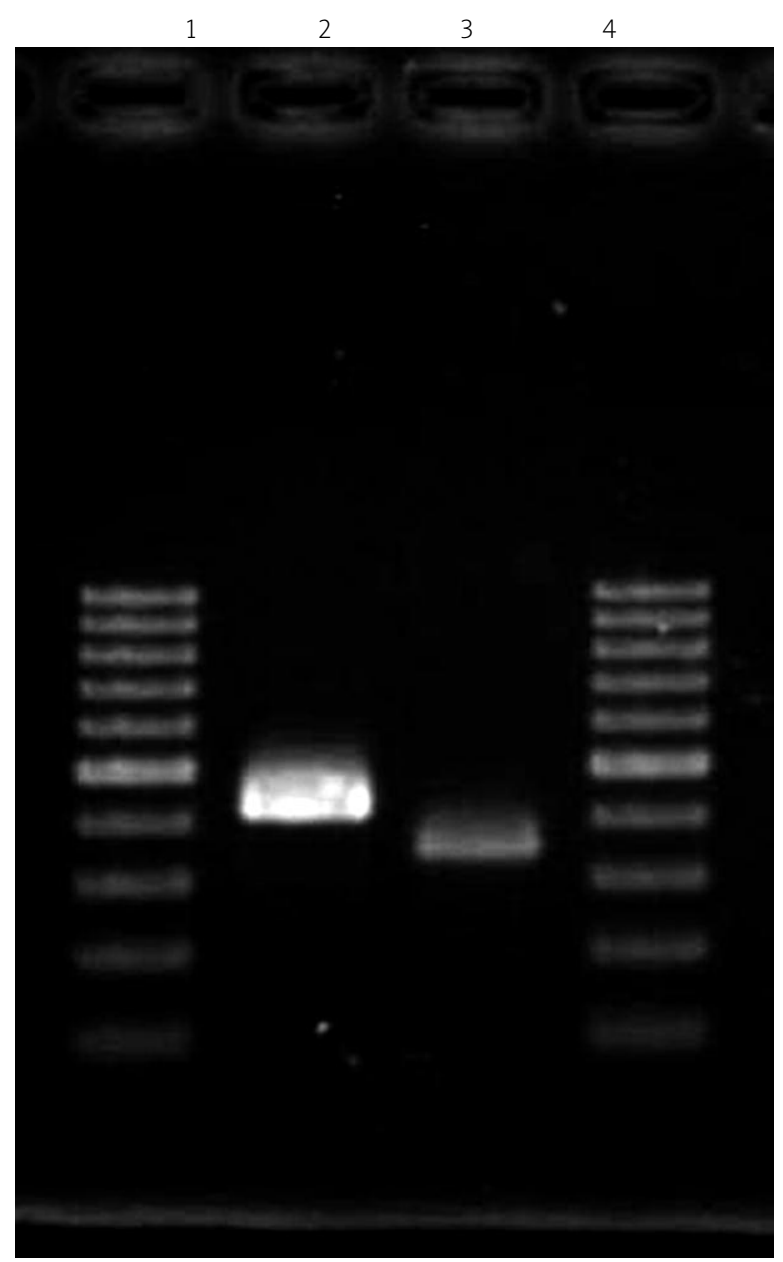

Fig. 2. $P C R$ results for $S$. aureus enterotoxin $A$ and C. difficile cytotoxin $B$. Lanes 1 and $4: 1 \mathrm{~kb}$ ladder; lanes 2 and 3 refer to $\operatorname{tcd} B$ and saeA, respectively 

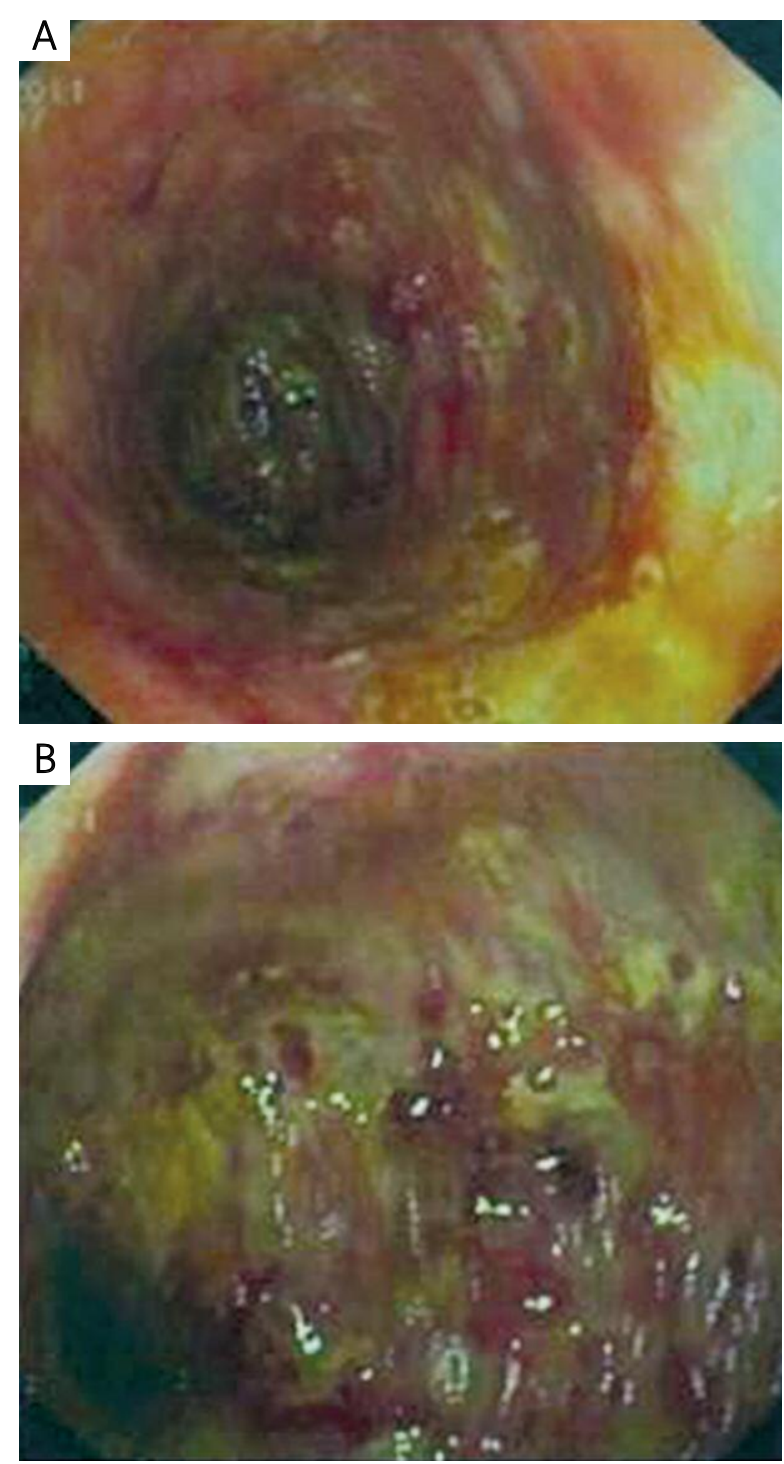

Fig. 3. Enteroscopy and sigmoidoscopy showed diffuse continuous erythema with diffuse ulceration in the colon $(\mathrm{A})$ that started at the rectum and stopped at the ascending colon (B). The mucosa was completely fragile with spontaneous bleeding

biotic usage suggested probable intestinal infection with drug-resistant bacteria, which could possibly explain his severe diarrhea. According to antibiogram results for
S. aureus (Table I), oral vancomycin (2 g daily) was started and was continued for 5 days. After initiation of the treatment, his symptoms were significantly relieved with nearly 3 defecations per day and his laboratory data showed improvement of the clinical conditions. In his colonoscopy there was diffuse ulceration in the colon that started at the rectum and terminated at the ascending colon (Figure 3). Also there were significant decreases in vascular marking and the mucosa was completely fragile with spontaneous bleeding. There was no mass or stricture during the examination. The terminal ileum had normal appearance. Sections from the sigmoid show an irregular luminal border with distorted glandular architecture, focal regenerative epithelial changes with severe diffuse infiltration of lymphoplasma cells. High numbers of eosinophils and neutrophils were present at the lamina propria as an index of active diffuse colitis. Multiple biopsies were obtained together with a new request for a stool exam. The result of culture was negative for the previous identified microorganisms. The bleeding started again after 5 days and symptoms were attributed to the IBD, instead of infectious colitis. The liver function tests showed worsening of liver activity. Because of a poor response to the maximum dose of the chosen drugs and deterioration of the clinical state of the patient, surgical consultation was requested and the surgeons decided to operate on the patient. The patient underwent a total colectomy and ileoanal anastomosis. Administration of imipenem $(500 \mathrm{mg}$, four times a day) and vancomycin (2 g daily) was started, but he did not respond to them. Gradually he was complicated with acute respiratory distress syndrome (ARDS) and also oliguria, and finally he expired with a diagnosis of septic shock a week after surgery. Severe infection during the disease with superantigenic $S$. aureus in conjunction with cytotoxic $C$. difficile bacteria was considered as a putative causative factor for failure in the disease management.

\section{Discussion}

Superimposed infections in patients with IBD have drawn considerable attention in the disease managements that can lead to increased rates of colectomy and mortality. This association was shown in some studies in the case of infections by $C$. difficile [8]. Staphylococcus aureus infection occasionally may occur during the

Table I. Frequency of resistance to antibiotics of $S$. aureus isolated from patient with IBD

\begin{tabular}{|c|c|c|c|c|c|c|c|c|c|c|c|c|}
\hline \multirow[t]{2}{*}{ Bacteria } & \multicolumn{12}{|c|}{ Antibiotics } \\
\hline & $P$ & $O x$ & $E$ & VAN & AN & SXT & $C$ & $T$ & GM & Cip & Lz & $\mathrm{Cc}$ \\
\hline S. aureus & $R$ & $R$ & $R$ & $S$ & $R$ & $R$ & $S$ & $R$ & $\mathrm{R}$ & $\mathrm{R}$ & $S$ & $S$ \\
\hline
\end{tabular}


Table II. Clinical findings and laboratory test results

\begin{tabular}{|c|c|c|c|c|c|c|c|c|c|c|c|c|c|c|c|}
\hline \multirow{2}{*}{ 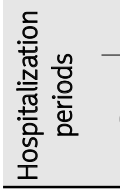 } & \multicolumn{15}{|c|}{ Tests } \\
\hline & $\begin{array}{l}\text { WBC } \\
\left(\times 10^{3}\right)\end{array}$ & $\begin{array}{c}\text { PMN } \\
{[\%]}\end{array}$ & $\begin{array}{c}\text { Plt } \\
\left(\times 10^{3}\right)\end{array}$ & $\begin{array}{l}\text { SGOT } \\
\text { [IU/I] }\end{array}$ & $\begin{array}{l}\text { SGPT } \\
{[I U / I]}\end{array}$ & $\begin{array}{c}\text { ALP } \\
{[I U / I]}\end{array}$ & $\begin{array}{c}\text { Bilirubin } \\
\mathrm{T} \\
{[\mathrm{mg} / \mathrm{dl}]}\end{array}$ & $\begin{array}{c}\text { Bilirubin } \\
\text { D } \\
\text { [mg/dl] }\end{array}$ & BUN & Creatinine & CRP & $\mathrm{BP}$ & PR & $\mathrm{T}$ & $\mathrm{RR}$ \\
\hline $\begin{array}{l}\text { Admis- } \\
\text { sion } \\
\text { time }\end{array}$ & 9.1 & 92.8 & 135 & 31 & 14 & 130 & 0.8 & 0.2 & 19 & 1.9 & 63 & $110 / 80$ & 80 & 37.3 & 12 \\
\hline $\begin{array}{l}\text { During } \\
\text { antibiotic } \\
\text { therapy }\end{array}$ & 9 & 90 & 107 & 37 & 34 & 488 & 0.9 & 0.3 & 16 & 1.2 & 28 & $200 / 90$ & 90 & - & 12 \\
\hline $\begin{array}{l}\text { After } \\
\text { surgery }\end{array}$ & 3.1 & 94 & 35 & 45 & 50 & 696 & 7 & 3.5 & 99 & 2 & - & $85 / 50$ & 131 & 37 & 16 \\
\hline
\end{tabular}

WBC - white blood cells, Plt - platelets, SGOT - serum glutamic-oxaloacetic transaminase, SGPT - serum glutamic-pyruvic transaminase, ALP - alkaline phosphatase, $B U N$ - blood urea nitrogen, $C R P-C$-reactive protein, $B P$ - blood pressure, $P R$ - pulse rate, $T$ - temperature, $R R$ - respiratory rate

Table III. List of primers used for screening of genes $t c d B$ and sea

\begin{tabular}{llccc} 
Bacteria & Oligonucleotide sequences & $\begin{array}{c}\text { Annealing } \\
\text { temperature }\left[{ }^{\circ} \mathrm{C}\right]\end{array}$ & $\begin{array}{c}\text { Length } \\
{[\mathrm{bp}]}\end{array}$ & References \\
\hline S. aureus sea & $\begin{array}{l}\text { F- ATGGTTATCAATGTGCGGGTGIIIIICCAAACAAAAC } \\
\text { R- TGAATACTGTCCTTGAGCACCAIIIIIATCGTAATTAAC }\end{array}$ & 55 & 344 & 17 \\
\hline C. difficile tcdB & F- GAGCTGCTTCAATTGGAGAGA & & & 18
\end{tabular}

course of IBD [9]. However, the potential role of its infection and virulence factors in the pathogenesis of IBD is not known. SAgs, such as staphylococcal enterotoxins, can induce $T$ cell proliferation. T cells are believed to be involved intimately in the regulation of the immune function in patients with IBD [7]. The SAgs can increase proinflammatory cytokine production from both healthy and inflamed colonic mucosa, suggesting that SAgs could be important initiators of the inflammatory cascade through direct T-cell activation, causing a massive cytokine release, which may end in shock. Sepsis could be the second complication caused by the suppressive effect of latter developed Treg cells on proliferation of naive peripheral blood mononuclear cells (PBMC) in response to bacterial infections. However, in a clinical setting it is difficult to find direct evidence for these involvements. Long duration of disease in patients with $I B D$, presence of inflamed mucosa together with chronic immunosuppressive medications have predisposed their intestines to infections with bacteria and other pathogens. Overgrowth of superantigenic bacteria in these patients could be problematic. In the present case, enormous numbers of superantigenic $S$. aureus with low numbers of cytotoxigenic isolates of $C$. difficile were detected in the patient's fecal sample. Decrease in numbers of defecations was a result of oral administration of vancomycin (the only effective antibiotic accord- ing to the laboratory antibiogram results), which suggested its involvement in disease severity. The $C$. difficile isolate was $t c d A$ negative and $t c d B$ positive. Potential cytotoxic activity of the $t c d B$ could be considered as an ancillary factor in the disease worsening. Comparison of the patient's vital signs and laboratory test results for WBC and platelet counts, liver enzymatic activities, and bilirubin, albumin, CRP, BUN, and creatinine levels during his hospitalization confirmed improvement of his enteric conditions for one week and success of vancomycin treatment in decreasing the CRP and BUN levels (Table II). This regime was not effective to control the increased liver enzymes during the study. Relapse of the disease with bleeding and rise in the noted values with no evidence of infection was the cause of the decision for surgery. Occurrence of septic shock, with the relative criteria, including temperature lower than $36^{\circ} \mathrm{C}$, heart rate greater than 90 beats/min, respiratory rate greater than 20 breaths/min or $\mathrm{PCO}_{2}$ lower than $32 \mathrm{~mm} \mathrm{Hg}$, WBC count lower than $4 \times 10 \% / /$ in conjugation with evidence of infection and oliguria, alteration of mental state, malfunction of vital organs and hypotension, were confirmed in this case after the surgery and did not change until the patient's expiration date (Table II). The IBD patients with unsatisfactory antibiotic therapy, such as ceftriaxone in our case, are under risk of antibiotic associated diarrhea and disease flare-up through the 
overgrowth of resistant bacteria in their intestines (e.g. C. difficile and S. aureus). Release of the overgrown intestinal $S$. aureus superantigens in high amounts and access of immune cells to them through the inflamed ulcerative mucosa was hypothesized as the major risk factor responsible for the patient's death after infection during the surgery. Immune cell depletion or suppression of PBMC by the proliferated T cells in response to these superantigens after initial exposure seems to be the reason for predisposing the patient to serious infections during the surgery that did not respond to the noted treatments. Increased PMN count to more than 94\% (Table II) at the time of surgery also supports a decrease in blood lymphocyte population, indirectly. There are some reports indicating the involvement of $S$. aureus superantigens in occurrence of sepsis and septic shock; however, no study has followed the suppressive effect of such antigens on the outcome of immune responses for predisposing their hosts to microbial superinfections and sepsis as a result of immunodeficiency. Association of $S$. aureus superantigens with IBD has been suggested, as lumen-derived $S$. aureus superantigens have been shown to elicit inflammation in a mouse model [10]. Also, the colon may serve as a reservoir of resistant S. aureus strains that were obtained during the patient's hospitalization. Usage of immunosuppressive drugs, such as azathioprine, that are used to inhibit the immune system in IBD patients, may leave the body more susceptible to infection by such pathogenic bacteria. The existing data in the present study only suggest such associations and establishment of this hypothesis needs more detailed studies. Nevertheless, coexistence of cytotoxigenic bacteria (e.g. C. difficile) and overgrown superantigenic $S$. aureus strains in the inflamed mucosa of IBD patients should be considered among the hospitalized patients. It is just as important to advise healthcare staff to report promptly should a sign of infection occur among these patients. Furthermore, to prevent overgrowth of life-threatening toxigenic or superantigenic resistant bacteria in the patients' intestine and minimize the complications, administration of antibiotics should be only considered if there is strong evidence of infection.

\section{Acknowledgments}

This study was supported by a grant from the Gastroenterology and Liver Diseases Research Center and the Basic and Molecular Epidemiology of Gastrointestinal Disorders Research Center, Shahid Beheshti University of Medical Sciences, Tehran, Iran. We would like to thank Dr. Mahsa Molaei and all of our staffs for their cooperation in this study.

\section{References}

1. Ogunbi SO, Ransom JA, Sullivan K, et al. Inflammatory bowel disease in African-American children living in Georgia. J Pediatr 1998; 133: 103-7.

2. Reuter BK, Pizarro TT. Commentary: the role of the IL-18 system and other members of the IL-1R/TLR superfamily in innate mucosal immunity and the pathogenesis of inflammatory bowel disease: friend or foe? Eur I Immunol 2004; 34: 2347-55.

3. Stallmach A, Carstens O. Role of infections in the manifestation or reactivation of inflammatory bowel diseases. Inflam Bowel Dis 2002; 8: 213-8.

4. Geboes K, De Hertogh G, Geboes KP. Inflammatory versus infectious bowel disease: more than a diagnostic dilemma. In: IBD year book. Bianchi Porro G, Ardizzone S, Colombo E (eds.). Nomos Edizioni, Milano 2004; 39-56.

5. Kelly D, Conway S, Aminov R. Commensal gut bacteria: mechanisms of immune modulation. Trends Immunol 2005; 26: 326-33.

6. Ouwehand A, Isolauri E, Salminen S. The role of the intestinal microflora for the development of the immune system in early childhood. Eur J Nutr 2002; 41: 132-7.

7. Rakoff-Nahoum S, Medzhitov R. Role of the innate immune system and host-commensal mutualism. Curr Top Microbiol Immunol 2006; 308: 1-18.

8. Kaufmann SH, Schaible UE. Antigen presentation and recognition in bacterial infections. Curr Opin Immunol 2005; 17 : 79-87.

9. Li, H, Llera A, Malchiodi EL, Mariuzza RA. The structural basis of T cell activation by superantigens. Annu Rev Immunol 1999; 17: 435-66.

10. Heriazon A, Zhou P, Borojevic R, et al. Regulatory T cells modulate staphylococcal enterotoxin B-induced effector T-cell activation and acceleration of colitis. Infect Immun 2009; 77: 707-13. 